\title{
Role of Electronic Relaxation Rates in the Casimir Force between High- $T_{c}$ Superconductors
}

\author{
Shunashi G. Castillo-López (1), Carlos Villarreal (), Giuseppe Pirruccio and Raúl Esquivel-Sirvent *
}

Citation: Castillo-López, S.G.;

Villarreal, C.; Pirruccio, G.;

Esquivel-Sirvent, R. Role of Electronic

Relaxation Rates in the Casimir Force between High- $T_{c}$ Superconductors.

Universe 2021, 7, 69. https://doi.org/

10.3390/universe7030069

Academic Editor: Galina L.

Klimchitskaya

Received: 27 February 2021

Accepted: 11 March 2021

Published: 13 March 2021

Publisher's Note: MDPI stays neutral with regard to jurisdictional claims in published maps and institutional affiliations.

Copyright: (c) 2021 by the authors. Licensee MDPI, Basel, Switzerland. This article is an open access article distributed under the terms and conditions of the Creative Commons Attribution (CC BY) license (https:// creativecommons.org/licenses/by/ $4.0 /)$.
Instituto de Física, Universidad Nacional Autónoma de México, Apartado Postal 20-364, Mexico 01000, Mexico; shunashi@fisica.unam.mx (S.G.C.-L.); carlos@fisica.unam.mx (C.V.); piruccio@fisica.unam.mx (G.P.)

* Correspondence: raul@fisica.unam.mx

\begin{abstract}
We revisit the problem of the Casimir force between high- $T_{c}$ superconductors below and above the critical temperature for the superconducting transition. Ceramic superconductors exhibit a different temperature dependence of the reflectivity when switching from the normal to the superconducting state. We leverage this unique characteristic with respect to ordinary metals to claim that these kind of materials can prove useful as an alternative system where the long-standing discussion on the role of electronic relaxation can be addressed. Furthermore, we show that the two main damping mechanisms associated with free and mid-infrared electrons dominate at very distinct scales, meaning that they can be considered separately when the Casimir force is measured as a function of slab distance. This facilitates the experimental identification of the role of the two electronic relaxation contributions to the force.
\end{abstract}

Keywords: Casimir force; superconductivity; high- $T_{\mathcal{C}}$ superconductors

\section{Introduction}

The Casimir force between planar surfaces has been a topic of intense research since its inception in 1948 [1,2]. Its reformulation by Lifshitz in terms of fluctuating electrodynamics related the force with the macroscopic dielectric properties of the materials [3]. Measurements of the Casimir force have been made using atomic force microscopy [4-7], torsional balances [8,9] and microelectromechanical systems [10,11], as well as in a wide variety of materials such as metals [12], semiconductors [13,14] and change-phase materials [15].

To compare the experimental results with the predictions of Lifshitz, an accurate description of the dielectric function is needed. In the case of metals, the dielectric function is obtained from experimental data and extrapolated at low frequencies with a Drude model [16]. The Drude model describes the intraband transitions, in particular those where the d-bands interact with nearly-free electron bands such as noble metals and alkali metals [17]. Two parameters define the Drude model: the plasma frequency, $\omega_{p}$, and the electronic relaxation rate, $\gamma_{p}$. Its use and accuracy describing many phenomena such as plasmonic excitation in noble metal nanoparticles $[18,19]$ or the D.C. conductivity of metals are well established, and its application to the Casimir problem should be straightforward. However, several studies aiming to measure Casimir forces in metals indicate that Lifshitz theory gives a better description of the experimental data if the damping term is omitted, and, instead, a simple plasma model is considered. Furthermore, it has been argued that the Nernst heat-theorem is not satisfied for Drude metals [20], but this has been disputed by some authors $[21,22]$. From a theoretical point of view, this statement is troublesome, since at zero-frequency the DC conductivity is finite in metals, as predicted by the Drude model. An extensive description of the problem and a possible resolution using the nonlocal response of the materials was recently proposed by Klimchitskaya and Mostepanenko [23].

As mentioned above, experiments reporting a better agreement with the plasma model have been performed by several groups. Recently, the Casimir force was measured using isoelectronic materials to reduce electrostatic effects [24]. On the other hand, the experiment 
of Sushkov et al. [25] measured the Casimir force in the range of $0.7-7 \mu \mathrm{m}$ and in this particular case the plasma model was excluded, giving rise to a discussion on the many issues that can affect the experiment such as patch potentials [26,27].

Another scenario of interest is the investigation of thermal properties of Casimir forces in setups involving either BCS or high- $T_{c}$ superconductors [28-31]. By considering a superconducting material such $\mathrm{NbTiN}$, with a critical temperature $T_{\mathcal{c}}=9.2 \mathrm{~K}$, Bimonte [28] calculated the force associated with changes in the temperature. Later on, a more detailed analysis of the dielectric function of type-I superconductors at imaginary frequencies was performed using the Mattis-Bardeen theory of superconductors [29].

Two experimental proposals exist related to the Casimir effect in BCS superconductors. In one case, involving a nanoelectromechanical system with a Pb layer deposited on a Au substrate, Perez-Morelo et al. [32] proposed that the Casimir energy would provoke a shift on $T_{c}$; however, no changes were observed for temperatures above $12 \mu \mathrm{K}$. On the other hand, Norte and coauthors [33] presented an on-chip platform including an optomechanical cavity to measure the Casimir effect between two closely-spaced freestanding superconductors as they transit into a superconducting state. Bimonte [30] showed that when working with $\mathrm{Al}$ the sensitivity of this device was not enough to detect changes in the Casimir force near $T_{c}$. However, the employment of $\mathrm{Al}$ and $\mathrm{NbTiN}$ could give enough contrast to detect changes in the force.

The issue of sensitivity can be alternatively addressed by considering high-temperature superconductors (HTSCs). A theoretical study was presented by Villarreal et al. [31] for optimally-doped $\mathrm{YBa}_{2} \mathrm{Cu}_{3} \mathrm{O}_{6.95}(\mathrm{YBCO})$ slabs, showing that in the long-distance regime a significant abrupt change in the Casimir force could be observed at the critical temperature. The dielectric function of YBCO considered in that work has three main contributions with different origins: one Drude-like from free electrons, a second mid-infrared (MIR) term associated with absorption of electromagnetic energy putatively due to electronic interband transitions, and several phonon contributions.

In this paper, we further explore the repercussion of relaxation phenomena in the Casimir effect. With that purpose, we revisit the Casimir force between YBCO superconductors as a function of plate separation in the short- $(L \sim 10-500 \mathrm{~nm})$ and long-distance $(L \sim 500-7000 \mathrm{~nm})$ regimes explored in previous experimental studies. We discuss the role of the electronic damping associated with either Drude or MIR contributions on the Casimir effect both in the normal and superconducting states.

\section{Formalism}

Consider two semi-infinite parallel plates of YBCO kept at the absolute temperature $T$ and separated by a vacuum gap of width $L$. Within the framework of Lifshitz theory, the finite-temperature force per unit area, $F(L, T)$, acting on both plates is described by the formula $[34,35]$

$$
F(L, T)=-\frac{k_{B} T}{\pi} \sum_{\ell=0}^{\prime} \int_{0}^{\infty} d \beta \beta \kappa_{0}\left(i \zeta_{\ell}, \beta\right) \sum_{\nu=s, p}\left\{\frac{\exp \left[2 \kappa_{0}\left(i \zeta_{\ell}, \beta\right) L\right]}{r_{\nu}^{2}\left(i \zeta_{\ell}, \beta\right)}-1\right\}^{-1} .
$$

Each term in Equation (1) represents the contribution of the $\ell$ th imaginary Matsubara frequency $i \zeta_{\ell}=i\left(2 \pi k_{B} T / \hbar\right) \ell$. The prime at the sum-symbol means that the $\ell=0$ term must be halved. In turn, the sum over $v$ accounts for the $s$ and $p$ polarization states of the electromagnetic radiation. The reflection coefficients for $s$ - and $p$-polarized waves impinging upon the (vacuum I YBCO) interface are given by the Fresnel equations

$$
r_{s}\left(i \zeta_{\ell}, \beta\right)=\frac{\kappa_{0}\left(i \zeta_{\ell}, \beta\right)-\kappa\left(i \zeta_{\ell}, \beta\right)}{\kappa_{0}\left(i \zeta_{\ell}, \beta\right)+\kappa\left(i \zeta_{\ell}, \beta\right)}, \quad r_{p}\left(i \zeta_{\ell}, \beta\right)=\frac{\varepsilon\left(i \zeta_{\ell}\right) \kappa_{0}\left(i \zeta_{\ell}, \beta\right)-\kappa\left(i \zeta_{\ell}, \beta\right)}{\varepsilon\left(i \zeta_{\ell}\right) \kappa_{0}\left(i \zeta_{\ell}, \beta\right)+\kappa\left(i \zeta_{\ell}, \beta\right)} .
$$


Using the imaginary frequency representation, the out-plane component of the wave vector inside the vacuum gap, $\kappa_{0}$, and within $\mathrm{YBCO}$ plate characterized by the permittivity $\varepsilon\left(i \zeta_{\ell}\right), \kappa$, are related to the in-plane component, $\beta$, by the expressions

$$
\kappa_{0}\left(i \zeta_{\ell}, \beta\right)=\sqrt{\frac{\zeta_{\ell}^{2}}{c^{2}}+\beta^{2}} \quad \text { and } \quad \kappa\left(i \zeta_{\ell}, \beta\right)=\sqrt{\varepsilon\left(i \zeta_{\ell}\right) \frac{\zeta_{\ell}^{2}}{c^{2}}+\beta^{2}} \text {. }
$$

The evaluation of the Casimir force (1) requires knowledge of the YBCO dielectric function $\varepsilon\left(i \zeta_{\ell}\right)$ in the normal and superconducting phases. To characterize the optical response of high- $T_{c}$ superconductors, two important questions must be addressed. First, HTSCs are layered materials with a highly anisotropic optical response. Second, for temperatures below $T_{c}$, the total charge density, $n$, is divided accordingly with London's two-fluid model into normal and SC contributions: $n=n_{n}(T)+n_{S}(T)$ [36]. When complemented with a Drude representation of HTSCs dielectric permittivity, discussed below, London' s model gives an account not only of the most salient electromagnetic features of these materials, such as the expulsion of magnetic field lines from their interior or an infinite DC conductivity, but also provides an accurate representation of their optical response. It is worth commenting that, in the case of BCS superconductors, the Mattis-Bardeen dielectric function [37] provides a better description of their optical properties as compared with the two-fluid model [30]. This holds in the so-called dirty limit, defined by $l<\xi_{0}$, being $l$ the mean free path and $\xi_{0}$ the zero-temperature coherence length of Cooper pairs. However, HTSC satisfy the clean-limit condition $l \gg \xi_{0}$. It is straightforward to show that in this limit the Mattis-Bardeen function reduces to Drude dielectric function.

The unit cell of $\mathrm{YBa}_{2} \mathrm{Cu}_{3} \mathrm{O}_{7-\delta}$ defines a uniaxial crystal, where the superfluid current is predominantly confined within two $\mathrm{CuO}_{2}$ planes, the $a b$-planes, equidistant from the central yttrium atom. In the transverse $c$-axis direction, charge transport mainly occurs due to interplane incoherent hopping; however, superfluid transport is also observed to a small extent near optimal doping $(\delta=0.05)$ [38]. The dielectric response of $\mathrm{YBa}_{2} \mathrm{Cu}_{3} \mathrm{O}_{6.95}$ with $T_{c}=93 \mathrm{~K}$ is thus specified by a diagonal dielectric tensor $\bar{\varepsilon}=\operatorname{diag}\left(\varepsilon_{a b}, \varepsilon_{a b}, \varepsilon_{c}\right)[39,40]$, where its components have been characterized through a number of experimental studies [38,41-45]. Explicit calculations incorporating the resulting parameterizations for $\varepsilon_{a b}(\omega)$ and $\varepsilon_{c}(\omega)$ show that the $c$-axis optical response leads to negligible contributions in the evaluation of frequency-integrated quantities, such as the heat flux between YBCO plates at different temperatures [46] or the Casimir force per unit area considered in this work. Therefore, to ease the interpretation of the derived results, in the following, we limit our analysis by contemplating only the $a b$-plane permittivity. In the normal regime, at $T>T_{\mathcal{C}}$, the measured optical spectra have been parameterized by a Drude-Lorentz dielectric function, expressed at imaginary frequencies as follows:

$$
\varepsilon_{n}\left(i \zeta_{\ell}\right)=\varepsilon_{\infty}+\frac{\omega_{p}^{2}}{\zeta_{\ell}^{2}+\gamma_{p} \zeta_{\ell}}+\frac{\Omega_{m i r}^{2}}{\zeta_{\ell}^{2}+\omega_{m i r}^{2}+\Gamma_{m i r} \zeta_{\ell}}+\sum_{j=1}^{6} \frac{S_{j} \omega_{p h, j}^{2}}{\zeta_{\ell}^{2}+\omega_{p h, j}^{2}+\gamma_{p h, j} \zeta_{\ell}} .
$$

The different terms represent, respectively, a high-frequency contribution, a Drudelike component due to free charge carriers, a mid-infrared Lorentz term, and six additional phonon contributions $[38,41,42,45]$. The values of the parameters at $T=100 \mathrm{~K}$ are given by $\varepsilon_{\infty}=3.8, \omega_{p}=0.75 \mathrm{eV}, \gamma_{p}=0.037 \mathrm{eV}, \Omega_{\text {mir }}=2.6 \mathrm{eV}$, $\omega_{\text {mir }}=0.26 \mathrm{eV}$, and $\Gamma_{\text {mir }}=1.0 \mathrm{eV}$. The phonon parameters $S_{j}, \omega_{p h, j}$ and $\gamma_{p h, j}$ are presented in [31]. This parameterization of the Drude band corresponds to a polycrystalline YBCO sample with $T_{c}=93 \mathrm{~K}$, and no significant variations in our results are expected with the different values reported for $\gamma_{p}$ at $T=100 \mathrm{~K}$ [41].

Concerning the second point, we assume that, even if the pairing mechanism in HTSCs is uncertain [47], superfluid properties may be described in terms of a 2D gas of weaklyinteracting Cooper-like pairs condensing at $T=T_{c}$. The dispersive properties of a dilute boson gas are described by an energy Bogoliubov spectrum $\mathcal{E}(p)=\left(p^{2} c_{S}^{2}+\left(p^{2} / 2 m\right)^{2}\right)^{1 / 2}$, 
where $p$ is the magnitude of transferred momentum and $c_{s}$ is the speed of sound. For sufficiently cold and dilute gases Bogoliubov spectrum reduces to a phonon dispersion relation: $\mathcal{E}(p) \rightarrow c_{s} p$. It can be shown that a $2 \mathrm{D}$ gas with this kind of spectrum satisfies [48]:

$$
\frac{\omega_{p s}^{2}(T)}{\omega_{p s}^{2}(0)}=\frac{n_{s}^{2}(T)}{n^{2}}=1-\frac{T^{2}}{T_{c}^{2}}
$$

where the SC plasma frequency is defined by $\omega_{p s}(T) \equiv n_{\mathcal{S}}(T) e^{2} / \varepsilon_{0} m$, for $T<T_{c}$. Similarly, the normal plasma frequency in this temperature regime is $\omega_{p n}(T) \equiv n_{n}(T) e^{2} / \varepsilon_{0} m$. Expression (5) provides a general and accurate representation of experimental measurements of the $a b$-plane penetration length $\lambda_{a b}(T)=c / \omega_{p s}(T)$ for a wide range of dopings of $\mathrm{YBa}_{2} \mathrm{Cu}_{3} \mathrm{O}_{7-\delta}$ samples [47].

In the SC regime, coherent charge transport is determined by the vanishing of the Drude relaxation parameter: $\gamma_{p} \rightarrow 0$. Additionally, in this work, we contemplate the possibility that the MIR-damping parameter also tends to the limit $\Gamma_{\text {mir }} \rightarrow 0$. The decrease of $\Gamma_{\text {mir }}$ would entail an increase in the intensity of the mid-infrared absorption band. The physical interpretation of this contribution is uncertain, but there is evidence indicating that even if this band is practically temperature independent, the damping parameter may significantly decrease when a single-crystalline sample is considered [41]. We address this question below within the framework of the Casimir force. Consequently, for $T<T_{\mathcal{C}}$, the dielectric function is described by

$$
\varepsilon_{s}\left(i \zeta_{\ell}\right)=\varepsilon_{\infty}+\frac{\omega_{p s}^{2}(T)}{\zeta_{\ell}^{2}}+\frac{\omega_{p n}^{2}(T)}{\zeta_{\ell}^{2}+\gamma_{p} \zeta_{\ell}}+\frac{\Omega_{m i r}^{2}}{\zeta_{\ell}^{2}+\omega_{m i r}^{2}+\Gamma_{m i r} \zeta_{\ell}}+\sum_{j=1}^{6} \frac{S_{j} \omega_{p h, j}^{2}}{\zeta_{\ell}^{2}+\omega_{p h, j}^{2}+\gamma_{p h, j} \zeta_{\ell}} .
$$

Assuming that the plasma frequency of the superconducting component does not vary at very low temperatures, we consider the measured value $\omega_{p s}(2 \mathrm{~K})=0.75 \mathrm{eV}$ and Equation (5) to evaluate the normal and superconducting contributions of the charge carriers in Equation (6). The values for $\Omega_{m i r}, \omega_{m i r}, \Gamma_{m i r}$ and $\varepsilon_{\infty}$ are the same as those obtained in the normal state. The phonon parameters are also very similar [31].

On the other hand, the effect of electronic relaxation is related to the contribution of the first $(\ell=0)$ Matsubara frequency in the force calculation (Equation (1)). To evaluate the zero-frequency limit of the reflection coefficients $r_{v}(0, \beta)$, we now introduce the expressions for normal (4) and superconducting (6) permittivities into Equation (2), obtaining

$$
\begin{gathered}
r_{s}(0, \beta)= \begin{cases}0 & \text { if } \quad \gamma_{p} \neq 0 \\
\frac{\beta-\sqrt{\omega_{p}^{2}+\beta^{2}}}{\beta+\sqrt{\omega_{p}^{2}+\beta^{2}}} & \text { if } \quad \gamma_{p}=0, \quad \text { for } T \geq T_{\mathcal{c}},\end{cases} \\
r_{s}(0, \beta)= \begin{cases}\frac{\beta-\sqrt{\omega_{p s}^{2}(T)+\beta^{2}}}{\beta+\sqrt{\omega_{p s}^{2}(T)+\beta^{2}}} & \text { if } \gamma_{p} \neq 0 \\
\frac{\beta-\sqrt{\omega_{p}^{2}+\beta^{2}}}{\beta+\sqrt{\omega_{p}^{2}+\beta^{2}}} & \text { if } \gamma_{p}=0, \quad \text { for } T<T_{\mathcal{c}},\end{cases}
\end{gathered}
$$

and

$$
r_{p}(0, \beta)=1, \quad \text { for all the above cases. }
$$

It is worth mentioning that the above limit expressions are valid regardless of the $\Gamma_{\text {mir }}$ value. In the following discussion, we employed the former expressions to evaluate the Casimir force per unit area in four different scenarios for temperatures either above or below $T_{\mathcal{C}}$ as a function of the temperature of the system and the YBCO plates separation $L$ : (i) $\gamma_{p} \neq 0, \Gamma_{\text {mir }} \neq 0$; (ii) $\gamma_{p} \neq 0, \Gamma_{\text {mir }}=0$; (iii) $\gamma_{p}=0, \Gamma_{\text {mir }} \neq 0$; and (iv) $\gamma_{p}=0, \Gamma_{\text {mir }}=0$. 


\section{Results}

We study the normalized Casimir force, $F / F_{0}$, between two semi-infinite $\mathrm{YBCO}$ slabs when both are in the normal, $T \geq T_{\mathcal{c}}$, or in the superconducting, $T<T_{\mathcal{c}}$, state. Here, $F_{0}=-\pi^{2} \hbar c / 240 L^{4}$ describes the Casimir force per unit area acting on two perfect conducting parallel plates at zero temperature. The effects of the relaxation rate of the free $\left(\gamma_{p}\right)$ and mid-infrared $\left(\Gamma_{\text {mir }}\right)$ electrons on the normalized force are investigated at the separation distances $L=10-500 \mathrm{~nm}$ and $L=500-7000 \mathrm{~nm}$. These are both relevant because typical experiments are performed in either the former or the latter case. Figure 1 shows the normalized force, $F / F_{0}$, as a function of the vacuum gap width, $L$. Solid curves correspond to the case of finite damping frequency, $\gamma_{p}=37 \mathrm{meV}$, i.e., modeling the free electrons contribution as a Drude term in the YBCO dielectric response (4). The normalized force predicted for a zero relaxation rate $\gamma_{p}=0$, which results from changing the Drude term in Equation (4) by the simpler plasma model $\omega_{p}^{2} / \zeta_{\ell}^{2}$, is shown with dashed curves. We also consider different values for the mid-infrared damping $\Gamma_{\text {mir }}=1$ or $0 \mathrm{eV}$, which can be distinguished by the color of the curves. Figure $1 \mathrm{a}, \mathrm{b}$ show that the effect of the mid-infrared damping $\Gamma_{m i r}$ is to lower $F / F_{0}$. On the other hand, it is clear that the relevance of $\gamma_{p}$ at short distances, $L=10-500 \mathrm{~nm}$, is practically negligible for both normal (Figure 1a) and superconducting (Figure $1 \mathrm{~b}$ ) plates. This can be explained by considering that the most significant contribution to the normalized Casimir force comes from those terms in the sum (1) with a frequency $\zeta_{\ell}$ close to the characteristic cavity frequency $\zeta_{c}=c / 2 L$. For small $L$, this characteristic frequency falls in the visible and infrared range $(\approx 9.8-0.2 \mathrm{eV})$, i.e., it is largely detuned from the relaxation rate values $\gamma_{p}=37$ or $0 \mathrm{meV}$. This has the important consequence that at short distances it is unfeasible to distinguish between a Drude- or a plasma-like behavior of the $\mathrm{YBCO}$ response. As $L$ increases, the lowest frequencies $\zeta_{\ell}$ in the sum Equation (1) acquire more importance, which implies that the effect of $\gamma_{p}$ on the normalized force becomes stronger. This can be appreciated by comparing the continuous and dashed (red or yellow) curves in Figure 1a,c, whose spacing increases with L. On the contrary, the influence of the mid-infrared term decreases, as indicated by the approaching of the (continuous or dashed) red and yellow curves. A similar argument holds for the superconducting state (Figure 1b,d), although with some important differences. For a temperature above the critical, the normalized Casimir force obtained using the Drude term with $\gamma_{p}=37 \mathrm{meV}$ displays a plateau in the range $L=500-7000 \mathrm{~nm}$, completely different from the nearly linear dependence predicted by the plasma model with $\gamma_{p}=0$ (see Figure 1c). The normalized force estimated by the simple plasma formula can be up to 1.5 times larger than that calculated with the Drude model, $F_{\text {plasma }}(100 \mathrm{~K}, 7000 \mathrm{~nm}) \approx 1.5 F_{\text {Drude }}(100 \mathrm{~K}, 7000 \mathrm{~nm})$. For superconducting plates, both models predict a similar behavior of the normalized force dependence with $L$, as well as similar magnitudes, being the maximum relative difference $\left[F_{\text {plasma }}(90 \mathrm{~K}, 7000 \mathrm{~nm})-F_{\text {Drude }}(90 \mathrm{~K}, 7000 \mathrm{~nm})\right] / F_{\text {Drude }}(90 \mathrm{~K}, 7000 \mathrm{~nm}) \approx 15 \%$.

For ease comparison and to further analyze the effect of the superconducting phase transition on the normalized Casimir force, some of the curves in Figure 1 are repeated in Figure 2. We limit the discussion to the case of $\Gamma_{\text {mir }}=1 \mathrm{eV}$ but similar observations hold for $\Gamma_{\text {mir }}=0$. For short distances (Figure 2a), the main contribution to the normalized force is provided by the temperature-independent mid-infrared band, which implies that the normal and the superconducting curves coincide. On the other hand, for large separations, the Drude model with $\gamma_{p}=37 \mathrm{meV}$ predicts a normalized force between superconducting plates up to $25 \%$ larger than that between two normal plates, $\left[F_{\text {Drude }}(90 \mathrm{~K}, 7000 \mathrm{~nm})-\right.$ $\left.F_{\text {Drude }}(100 \mathrm{~K}, 7000 \mathrm{~nm})\right] / F_{\text {Drude }}(100 \mathrm{~K}, 7000 \mathrm{~nm}) \approx 25 \%$ (see Figure $2 \mathrm{~b}$ ). Conversely, the dashed curves obtained using the plasma model are practically identical for normal and superconducting plates. The previous results are explained by recalling that, for large $L$, the most important contribution in the sum (1) is given by the zero-frequency reflectivities (7). Using the plasma model, the reflectivities $r_{s}(0, \beta)$ are the same in the normal and superconducting states, while, for the Drude model $r_{s}(0, \beta)=0$ in the normal state, and it is temperature-dependent in the superconducting phase. Therefore, accord- 
ing to Equations (7a) and (7b) in the case $\gamma_{p} \neq 0$, we expect an increased difference $F_{\text {Drude }}\left(T<T_{\mathcal{c}}, L\right)-F_{\text {Drude }}\left(T>T_{\mathcal{c}}, L\right)$ as the temperature decreases.
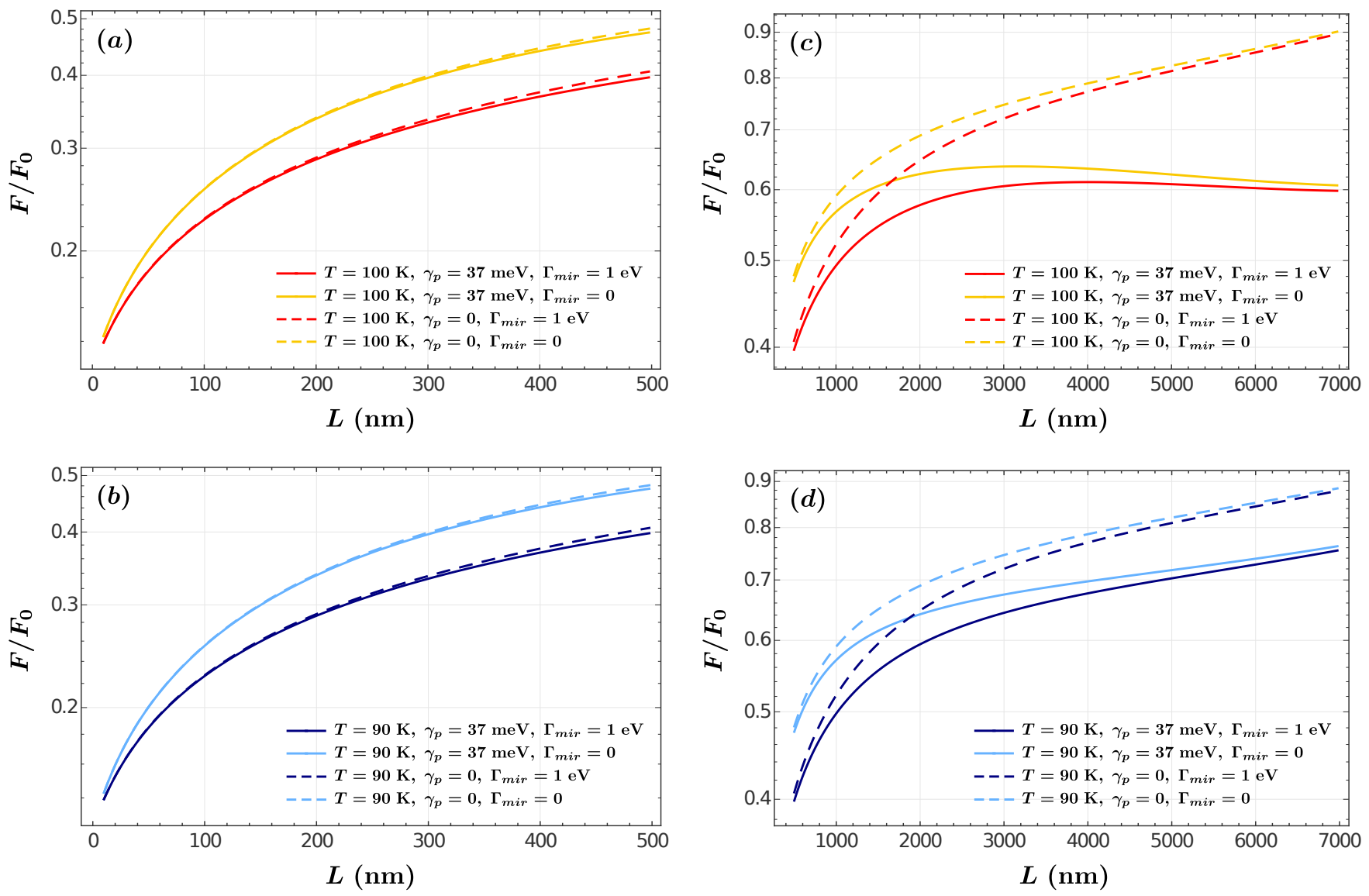

Figure 1. Normalized Casimir force, $F / F_{0}$, as a function of the vacuum gap width, $L$, for temperatures $T>T_{\mathcal{C}}(\mathbf{a}, \mathbf{c})$ and $T<T_{\mathcal{C}}(\mathbf{b}, \mathbf{d})$. Solid curves correspond to a free electrons relaxation rate $\gamma_{p}=37 \mathrm{meV}$, while the dashed ones to $\gamma_{p}=0$. Different colors refer to the value of the mid-infrared damping parameter $\Gamma_{\text {mir }}$.
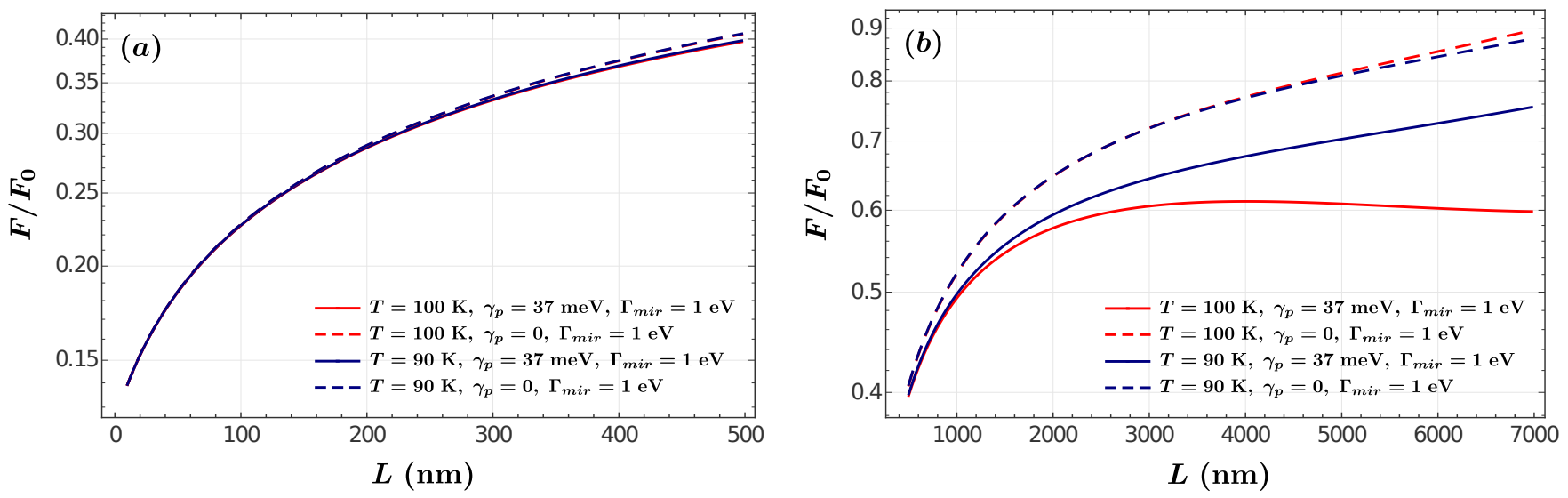

Figure 2. Comparison of the normalized Casimir force between normal $(T=100 \mathrm{~K})$ and superconducting $(T=90 \mathrm{~K}) \mathrm{YBCO}$ plates for short (a) and large (b) separation distances using $\gamma_{p}=37 \mathrm{meV}$ (solid line) and $\gamma_{p}=0$ (dashed line). In all cases. the mid-infrared damping $\Gamma_{\text {mir }}=1 \mathrm{eV}$.

\section{Conclusions}

We study the effects of free and mid-infrared electron relaxation rates on the Casimir force between two superconducting slabs made of optimally doped YBCO. The influence of these two damping mechanisms is analyzed for the case of both plates being below or 
above the critical temperature for the superconducting phase transition. The temperatureindependent mid-infrared damping causes a reduction in the normalized Casimir force and its influence decreases with the increase of the vacuum gap width. On the other hand, the effect of the free electrons damping is negligible at short separations for both normal and superconducting plates, while it dominates for distances in the micrometer scale. Here, the plasma model estimates a normalized force up to $50 \%(15 \%)$ larger than that obtained with the Drude model for two normal (superconducting) plates. Our results show that Drude model predicts an increase of up to $25 \%$ in the normalized force when both plates become superconducting, whereas, according to the plasma model, no significant changes in the normalized force are expected as a result of the phase transition. This indicates that high-temperature superconductors represent a valid alternative to ordinary metals to explore the role of damping in the Casimir force.

Author Contributions: S.G.C.-L. conducted the numerical calculations. All authors analyzed the results and contributed to the manuscript. All authors have read and agreed to the published version of the manuscript.

Funding: S.G.C-L. acknowledges support from CONACyT-Grant A1-S-10537. R.E.-S. acknowledges partial support from DGAPA-UNAM grant IN110-819. G.P. acknowledges the partial support from DGAPA-PAPIIT grant IN107319. All authors acknowledge the partial support from DGAPA-PAPIIT grant IN114920.

Data Availability Statement: Not applicable.

Conflicts of Interest: The authors declare no conflict of interest.

\section{References}

1. Casimir, H.B.G. On the attraction between two perfectly conducting plates. Proc. Akad. Wet. Amst. 1948, 51, 793-795.

2. Casimir, H.B.G.; Polder, D. The Influence of Retardation on the London-van der Waals Forces. Phys. Rev. 1948, $73,360-372$. [CrossRef]

3. Lifshitz, E.M. The theory of molecular attractive forces between solids. Sov. Phys. 1956, 2, 73.

4. Mohideen, U.; Roy, A. Precision Measurement of the Casimir Force from 0.1 to 0.9 microns. Phys. Rev. Lett. 1998, 81, 4549-4552. [CrossRef]

5. Roy, A.; Lin, C.Y.; Mohideen, U. Improved precision measurement of the Casimir force. Phys. Rev. D 1999, 60, 111101. [CrossRef]

6. Harris, B.W.; Chen, F.; Mohideen, U. Precision measurement of the Casimir force using gold surfaces. Phys. Rev. A 2000, 62, 052109. [CrossRef]

7. van Zwol, P.J.; Palasantzas, G.; De Hosson, J.T.M. Influence of random roughness on the Casimir force at small separations. Phys. Rev. B 2008, 77, 075412. [CrossRef]

8. Lamoreaux, S.K. Demonstration of the Casimir Force in the 0.6 to 6 micron Range. Phys. Rev. Lett. 1997, 78, 5-8. [CrossRef]

9. Garcia-Sanchez, D.; Fong, K.Y.; Bhaskaran, H.; Lamoreaux, S.; Tang, H.X. Casimir Force and In Situ Surface Potential Measurements on Nanomembranes. Phys. Rev. Lett. 2012, 109, 027202. [CrossRef] [PubMed]

10. Decca, R.S.; López, D.; Fischbach, E.; Klimchitskaya, G.L.; Krause, D.E.; Mostepanenko, V.M. Tests of new physics from precise measurements of the Casimir pressure between two gold-coated plates. Phys. Rev. D 2007, 75, 077101. [CrossRef]

11. Decca, R.S.; López, D.; Fischbach, E.; Krause, D.E. Measurement of the Casimir Force between Dissimilar Metals. Phys. Rev. Lett. 2003, 91, 050402. [CrossRef]

12. Banishev, A.A.; Klimchitskaya, G.L.; Mostepanenko, V.M.; Mohideen, U. Demonstration of the Casimir Force between Ferromagnetic Surfaces of a Ni-Coated Sphere and a Ni-Coated Plate. Phys. Rev. Lett. 2013, 110, 137401. [CrossRef] [PubMed]

13. Chen, F.; Mohideen, U.; Klimchitskaya, G.L.; Mostepanenko, V.M. Investigation of the Casimir force between metal and semiconductor test bodies. Phys. Rev. A 2005, 72, 020101. [CrossRef]

14. Chen, F.; Mohideen, U.; Klimchitskaya, G.L.; Mostepanenko, V.M. Experimental test for the conductivity properties from the Casimir force between metal and semiconductor. Phys. Rev. A 2006, 74, 022103. [CrossRef]

15. Torricelli, G.; van Zwol, P.J.; Shpak, O.; Binns, C.; Palasantzas, G.; Kooi, B.J.; Svetovoy, V.B.; Wuttig, M. Switching Casimir forces with phase-change materials. Phys. Rev. A 2010, 82, 010101. [CrossRef]

16. Svetovoy, V.B.; van Zwol, P.J.; Palasantzas, G.; De Hosson, J.T.M. Optical properties of gold films and the Casimir force. Phys. Rev. B 2008, 77, 035439. [CrossRef]

17. Deegan, R.A. Total Energy of $d$-Band Metals: Alkaline-Earth and Noble Metals. Phys. Rev. 1969, 186, 619-624. [CrossRef]

18. Maier, S. Plasmonics-Fundamentals and Applications; Springer: Berlin, Germany, 2007.

19. Davis, T.J.; Gómez, D.E. Colloquium: An algebraic model of localized surface plasmons and their interactions. Rev. Mod. Phys. 2017, 89, 011003. [CrossRef] 
20. Bezerra, V.B.; Klimchitskaya, G.L.; Mostepanenko, V.M.; Romero, C. Violation of the Nernst heat theorem in the theory of the thermal Casimir force between Drude metals. Phys. Rev. A 2004, 69, 022119. [CrossRef]

21. Høye, J.S.; Brevik, I.; Ellingsen, S.A.; Aarseth, J.B. Analytical and numerical verification of the Nernst theorem for metals. Phys. Rev. E 2007, 75, 051127. [CrossRef]

22. Bezerra, V.B.; Decca, R.S.; Fischbach, E.; Geyer, B.; Klimchitskaya, G.L.; Krause, D.E.; López, D.; Mostepanenko, V.M.; Romero, C. Comment on "Temperature dependence of the Casimir effect". Phys. Rev. E 2006, 73, 028101. [CrossRef] [PubMed]

23. Klimchitskaya, G.L.; Mostepanenko, V.M. An alternative response to the off-shell quantum fluctuations: A step forward in resolution of the Casimir puzzle. Eur. Phys. J. C 2020, 80, 900. [CrossRef]

24. Bimonte, G.; López, D.; Decca, R.S. Isoelectronic determination of the thermal Casimir force. Phys. Rev. B 2016, 93, 184434. [CrossRef]

25. Sushkov, A.; Kim, W.; Dalvit, D.; Lamoreaux, S. Observation of the thermal Casimir force. Nat. Phys. 2011, 7, 230-233. [CrossRef]

26. Behunin, R.; Dalvit, D.; Decca, R.; Speake, C. Limits on the accuracy of force sensing at short separations due to patch potentials. Phys. Rev. D 2014, 89, 051301. [CrossRef]

27. Behunin, R.O.; Intravaia, F.; Dalvit, D.A.R.; Neto, P.A.M.; Reynaud, S. Modeling electrostatic patch effects in Casimir force measurements. Phys. Rev. A 2012, 85, 012504. [CrossRef]

28. Bimonte, G. Casimir effect in a superconducting cavity and the thermal controversy. Phys. Rev. A 2008, 78, 062101. [CrossRef]

29. Bimonte, G.; Haakh, H.; Henkel, C.; Intravaia, F. Optical BCS conductivity at imaginary frequencies and dispersion energies of superconductors. J. Phys. A Math. Theor. 2010, 43, 145304. [CrossRef]

30. Bimonte, G. Casimir effect between superconductors. Phys. Rev. A 2019, 99, 052507. [CrossRef]

31. Villarreal, C.; Caballero-Benitez, S.F. Casimir forces and high-T c superconductors. Phys. Rev. A 2019, 100, 042504. [CrossRef]

32. Pérez-Morelo, D.; Stange, A.; Lally, R.W.; Barrett, L.K.; Imboden, M.; Som, A.; Campbell, D.K.; Aksyuk, V.A.; Bishop, D.J. A system for probing Casimir energy corrections to the condensation energy. Microsyst. Nanoeng. 2020, 6, 115. [CrossRef]

33. Norte, R.A.; Forsch, M.; Wallucks, A.; Marinković, I.; Gröblacher, S. Platform for Measurements of the Casimir Force between Two Superconductors. Phys. Rev. Lett. 2018, 121, 030405. [CrossRef]

34. Lambrecht, A.; Reynaud, S. Casimir force between metallic mirrors. Eur. Phys. J. D 2000, 8, 309-318. [CrossRef]

35. Mochán, W.L.; Villarreal, C. Casimir effect for arbitrary materials: Contributions within and beyond the light cone. New J. Phys. 2006, 8, 242. [CrossRef]

36. Annett, J.F. Superconductivity, Superfluids and Condensates; Oxford University Press: Oxford, UK, 2004 ; Volume 5.

37. Mattis, D.; Bardeen, J. Theory of the anomalous skin effect in normal and superconducting metals. Phys. Rev. 1958, 111, 412. [CrossRef]

38. Basov, D.N.; Timusk, T. Electrodynamics of high- $T_{c}$ superconductors. Rev. Mod. Phys. 2005, 77, 721-779. [CrossRef]

39. Biehs, S.A.; Ben-Abdallah, P.; Rosa, F.S.; Joulain, K.; Greffet, J.J. Nanoscale heat flux between nanoporous materials. Opt. Express 2011, 19, A1088-A1103. [CrossRef]

40. Stinson, H.; Wu, J.; Jiang, B.; Fei, Z.; Rodin, A.; Chapler, B.; McLeod, A.; Neto, A.C.; Lee, Y.; Fogler, M.; et al. Infrared nanospectroscopy and imaging of collective superfluid excitations in anisotropic superconductors. Phys. Rev. B 2014, $90,014502$. [CrossRef]

41. Timusk, T.; Herr, S.L.; Kamarás, K.; Porter, C.D.; Tanner, D.B.; Bonn, D.A.; Garrett, J.D.; Stager, C.V.; Greedan, J.E.; Reedyk, M. Infrared studies of ab-plane oriented oxide superconductors. Phys. Rev. B 1988, 38, 6683-6688. [CrossRef] [PubMed]

42. Bonn, D.A.; O’Reilly, A.H.; Greedan, J.E.; Stager, C.V.; Timusk, T.; Kamarás, K.; Tanner, D.B. Far-infrared properties of ab-plane

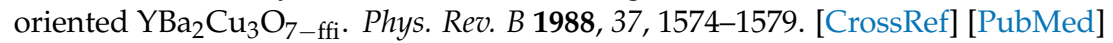

43. Homes, C.; Timusk, T.; Bonn, D.; Liang, R.; Hardy, W. Optical properties along the c-axis of YBa2Cu3O6+x, for $x=0.50 \rightarrow 0.95$ evolution of the pseudogap. Phys. C Supercond. 1995, 254, 265-280. [CrossRef]

44. Homes, C.; Timusk, T.; Bonn, D.; Liang, R.; Hardy, W. Optical phonons polarized along the c axis of YBa2Cu3O6+ $\mathrm{x}$, for $x=0.5 \rightarrow 0.95$. Can. J. Phys. 1995, 73, 663-675. [CrossRef]

45. Tanner, D.B.; Timusk, T. Optical properties of high-temperature superconductors. In Physical Properties of High Temperature Superconductors III; World Scientific Publishing Co.: Singapore, 1998; Chapter 5, pp. 363-469.

46. Castillo-López, S.; Pirruccio, G.; Villarreal, C.; Esquivel-Sirvent, R. Near-field radiative heat transfer between high-temperature superconductors. Sci. Rep. 2020, 10,1-10. [CrossRef]

47. Chen, Q.; Stajic, J.; Tan, S.; Levin, K. BCS-BEC crossover: From high temperature superconductors to ultracold superfluids. Phys. Rep. 2005, 412, 1-88. [CrossRef]

48. Lomnitz, M.; Villarreal, C.; De Llano, M. BEC model of high-Tc superconductivity in layered cuprates. Int. J. Mod. Phys. B 2013, 27, 1347001. [CrossRef] 